\title{
Resonancia Magnética Renal en un paciente con Hemoglobinuria Paroxística Nocturna
}

\author{
Liliam Escariz B. ${ }^{1}$, Cristina Pallasco M. ${ }^{2}$, Dorien Segers ${ }^{3}$, Víctor Chávez G. ${ }^{4}$, Katiuska Mederos M. ${ }^{5}$
}

1. Universidad Laica Eloy Alfaro, Manta, Manabí, Ecuador.

2. Licenciada en Radiología e Imagenología, Ecuador.

3. Universidad Laica Eloy Alfaro, Manta, Manabí, Ecuador.

4. Médico tratante en SOLCA, Portoviejo, Manabí, Ecuador.

5. Universidad Central Milagros, Milagros, Guayas, Ecuador.

\section{Renal Magnetic Resonance in a patient with paroxysmal nocturnal hemoglobinuria}

\begin{abstract}
Paroxysmal nocturnal hemoglobinuria $(P N H)$ is an acquired clonal disease causing hematopoietic cell alteration, caused by a mutation in the PIG-A gene; which does not allow certain important proteins to bind to the cells. A 58-year-old patient presented with headache, a history of malaria, and occasionally dark urine. Magnetic resonance imaging proved to be an effective diagnostic method for detecting renal cortical hemosiderosis, thanks to its high spatial resolution capacity. Keywords. Hemoglobinuria, Magnetic Resonance, Hematuria.
\end{abstract}

\section{Resumen}

La hemoglobinuria paroxística nocturna (HPN) es una enfermedad clonal adquirida ocasionando la alteración de células hematopoyética, causada por una mutación en el gen GPI-A; lo cual no permite que ciertas proteínas importantes se fijen a las células. Se presenta un paciente de 58 años que acude con cefalea, antecedente de malaria y en ocasiones orinas oscuras. La resonancia magnética demostró ser un método diagnóstico eficaz para detectar la hemosiderosis renal cortical, gracias a su elevada capacidad de resolución espacial.

Palabras claves. Hemoglobinuria, Resonancia Magnética, Hematuria.

Escariz L. Resonancia Magnética Renal en un paciente con Hemoglobinuria Paroxística Nocturna. Rev Chil Radiol 2017;23(2): 55-58.

Correspondencia: Dorien Segers./ doriensegers@hotmail.com

Trabajo recibido el 02 de abril 2017. ceptado para publicación el 13 de junio de 2017.

\section{Justificación}

La Hemoglobinuria Paroxística Nocturna (HPN) es una rara enfermedad adquirida que ocasiona una producción alterada de células sanguíneas y su descomposición rápida. Se produce por la alteración en el gen PIG-A de las células hematopoyéticas causando una deficiencia parcial o total del glucosilfosfatidilinositol, impidiendo que se fijen a la membrana proteínas importantes, que son las que protegen e inhiben el complejo de ataque a membrana'. La historia natural de la HPN es la hipersensibilidad de los eritrocitos al complemento intravascular que se traduce en crisis hemolíticas nocturnas en pacientes de 30 años, aunque la enfermedad también se puede presentar en ancianos y niños ${ }^{2}$. Las cifras de incidencia hablan de mayor frecuencia en países asiáticos, seguido por Europa, pero los casos son de 5 por cada 500.000 personas ${ }^{2}$. En Ecuador, su tasa anual de incidencia se desconoce ${ }^{4}$. Es necesaria la realización de una historia clínica detallada destinada a detectar la presencia de los síntomas y signos característicos que pueden ir de leves a graves, como la astenia, disfagia, fiebre, cólicos, dolor lumbar, disnea, cefaleas, orina oscura, fatiga, palidez e ictericia. Las complicaciones son la anemia aplásica, la leucemia aguda y los fenómenos tromboembólicos que pueden pasar inadvertidos, y es la principal causa de muerte. La supervivencia media de los pacientes se sitúa en torno a los 10-15 años ${ }^{4}$. 
La introducción de técnicas radiológicas como la ecografía y la resonancia magnética, junto a la sucesiva ampliación de sus aplicaciones diagnósticas ha supuesto una auténtica revolución dentro de la medicina ${ }^{4}$. La resonancia magnética es la técnica de elección, que muestra una inversión de la intensidad normal entre la cortical y la médula en T1 y una baja intensidad cortical en T2 producida por siderosis cortical renal. Siendo una prueba que permite evaluar claramente los órganos abdominales ${ }^{4}$.

Este trabajo investigativo pretende brindar un aporte útil a los profesionales de la salud, licenciados en imagenología y a la ciudadanía en general para que conozcan sobre la HPN, en el área de nefrología y el papel diagnóstico que ocupa la resonancia magnética sin contraste ni maniobras invasivas.

\section{Presentación del caso}

Paciente masculino de 58 años, ecuatoriano, piel morena, comerciante, de situación socioeconómica baja. Acude con cefalea, náuseas, con antecedentes personales de malaria y orinas oscuras en algunas ocasiones. Al examen físico muestra abdomen doloroso con irradiación lumbar, y encontramos ligera palidez cutaneomucosa e ictericia moderada. Las pruebas de laboratorio como hemograma, conteo leucocitario y el ionograma se mostraron normales.

La valoración radiológica pulmonar refleja un índice cardiotorácico normal. Sin condensación parenquimatosa pulmonar, ni opacidad nodular, su trama pulmonar está normal, ángulos costodiafragmáticos normales y sin ensanchamiento mediastinal. Dentro de los límites de normalidad.

La ecografía de abdomen superior refleja:

- Hígado de tamaño normal, bordes finos, superficie lisa, sin lesiones ocupativas de espacio

- Vesícula biliar: colecistectomizado.

- Vías biliares normales.

- Páncreas dentro de los parámetros normales.

- Riñones de tamaño normal, con adecuada diferenciación cortico-medular, sin litiasis, ni hidronefrosis.

- ID: Dentro de límites normales

Se le realiza colangioresonancia simple, reportando:

- Vías biliares extrahepáticas e intrahepáticas: calibre normal.

- Vena porta: $11 \mathrm{~mm}$.

- Venas hepáticas: De calibre normal.

- Vesícula: Colecistectomizado

- No se observa masa biliar ni hepática

- Aorta de calibre normal.

- No se observan nódulos linfáticos.

- Hígado: con medidas de $77 \mathrm{~mm}$ lóbulo izquierdo un y $159 \mathrm{~mm}$ lóbulo derecho, de patrón difuso hipointenso en T2 con aspecto ligero de siderosis.
- Bazo. De tamaño normal, mide $118 \mathrm{~mm}$. Espacio espleno-renal libre.

- Páncreas: de tamaño normal, cabeza mide $22 \mathrm{~mm}$ cuerpo 17 y cola $18 \mathrm{~mm}$.

- Glándulas Suprarrenales: De caracteres normales.

- Riñones: Derecho mide $12 \mathrm{~cm}$. parénquima de $23 \mathrm{~mm}$. Izquierdo mide $11.8 \mathrm{~cm}$ parénquima de $22 \mathrm{~mm}$.

En ambos se observa inversión del patrón de intensidad en T1 e hipointensidad en T2 de la corteza con aspecto de siderosis cortical. Signos de carga siderótica renal. Se encuentra como hallazgo casual el depósito de hierro en ambas cortezas renales.

\section{Impresión Diagnóstica: Hemoglobinuria Paroxística Nocturna.}

\section{Discusión}

La hemoglobinuria paroxística nocturna es una enfermedad clonal adquirida en las células hematopoyéticas, haciendo que el glicosilfosfatidil-inositol se ausente en la membrana celular, causando que no se fijen a la membrana proteínas importantes, las que protegen a la membrana del ataque del complemento. La hemoglobinuria paroxística nocturna no es una enfermedad congénita, sino adquirida en el transcurso de la vida, cuando se da una mutación y por lo tanto hay un clon celular ${ }^{5}$. La HPN es potencialmente grave y su curso clínico es impredecible: hay afectados que fallecen a unos cuantos meses del diagnóstico y otros de larga evolución ${ }^{6}$. La fisiopatología se relaciona con una hemólisis continuada, un fallo renal, una trombosis o incluso una leucemia aguda. El seguimiento de estos pacientes debe ser muy preciso.

Concordando con Ruiz et al. ${ }^{7}$, se considera a la RM como el único método de exploración que puede demostrar el depósito de hierro en la corteza renal y las complicaciones que puede acarrear. Además, puede determinar la concentración de hierro en los riñones, y evitar pasar por alto algún detalle no visto en otros exámenes de imagen. Tanaka, et al. ${ }^{8}$ concluyeron que en la $\mathrm{RM}$ el depósito de hierro en los riñones es un hallazgo muy característico de la hemoglobinuria paroxística nocturna, aunque estos depósitos pueden estar presentes en la médula y la corteza. La menor intensidad de señal se da en la corteza tanto en T1 y T2; lo que significa que la concentración de hierro en la corteza es mayor, signo patognomónico de la HPN.

Suzukawa et al. ${ }^{9}$ resaltan que una función deficiente de la médula ósea también puede contribuir a que no se produzca la cantidad normal de glóbulos rojos y que no tengan la capacidad de remplazar las células perdidas, siendo un factor que contribuye al desarrollo de una anemia en el paciente con HPN, con 


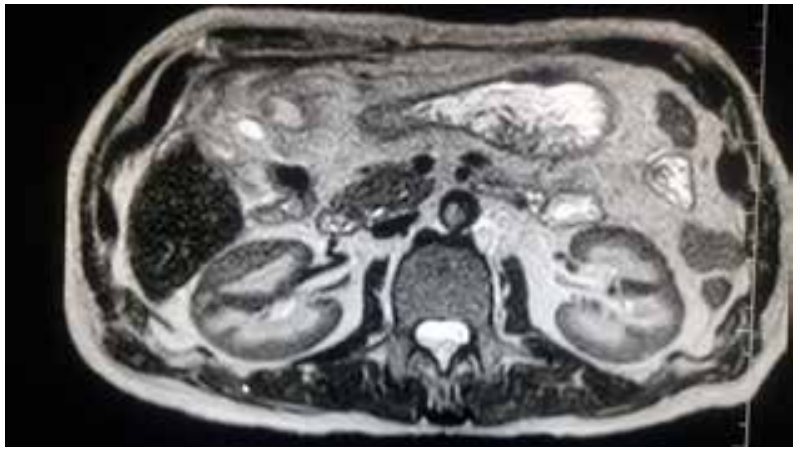

Figura 1. Corte axial de RM abdominal en T2, se observa la cortical de ambos riñones marcadamente hipointensa.

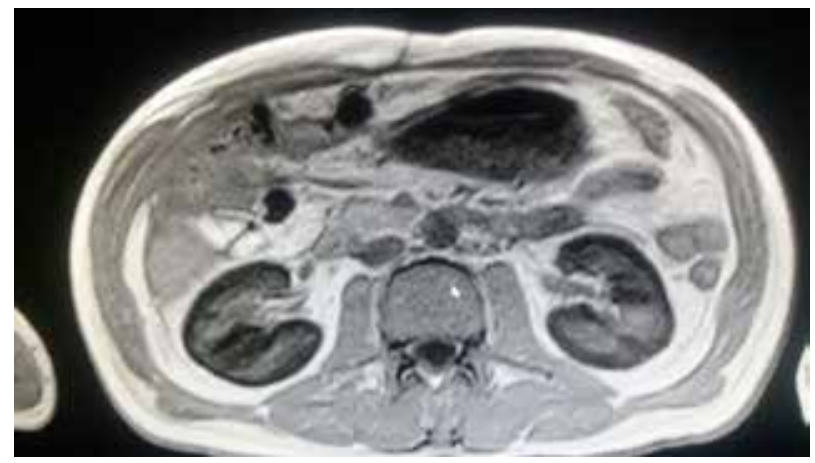

Figura 3. Corte axial en T1. La intensidad de señal de la corteza renal es inferior a la de la región medular.

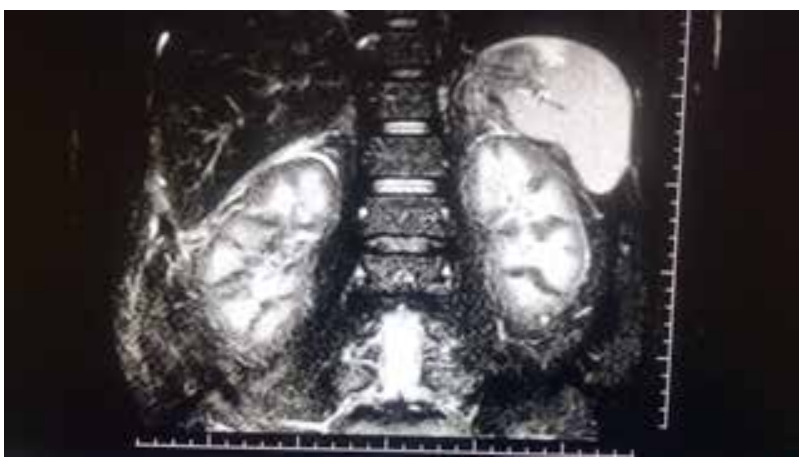

un recuento de reticulocitos inadecuadamente baja.

Seguimientos clínicos de pacientes con HPN, incluidos estudios necrópsicos, revelan la existencia de depósitos de hemosiderina en los túbulos proximales, también en casos de pacientes afectos de HPN que había cursado no severamente; algunos pueden desarrollar insuficiencia renal crónica como resultado de varios factores, como son los microinfartos por repetidos episodios de trombosis ${ }^{10-13}$.

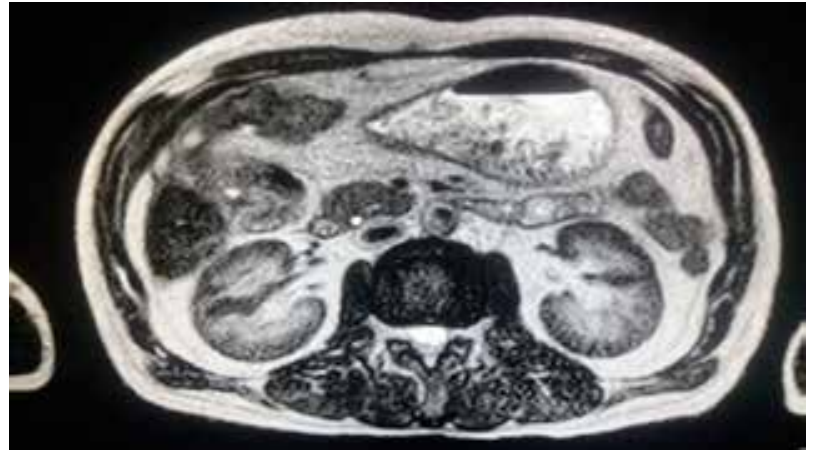

Figura 2. Corte axial en T2, muestra la diferencia de intensidad corteza-médula.

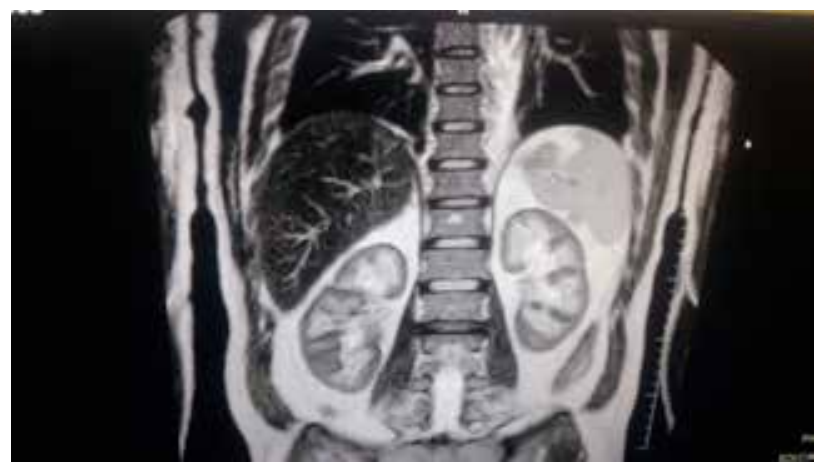

Figura 4. Corte coronal en T1 que evidencia una afectación cortical renal bilateral.

Figura 5. Corte coronal que evidencia una hipointensidad en la corteza de ambos riñones.

\section{Conclusiones}

Puede llegar a ser difícil detectar la HPN, por eso es necesario la realización de anamnesis, análisis de laboratorio, rayos $\mathrm{x}$, ecografía y resonancia magnética. En este último examen imagenológico se confirma esta enfermedad, mostrando la afectación renal como depósitos de hemosiderina en la corteza de ambos riñones.

Actualmente existen subregistros sobre esta en- 
fermedad, ni es frecuente en la práctica médica la resonancia magnética como herramienta diagnóstica de la HPN. Puede tardar años en ser diagnosticada, ya que es una enfermedad rara y se adquiere a lo largo de la vida, los pacientes pueden presentar síntomas o lo confunden con algo pasajero. Además de pruebas de laboratorio, es pertinente que a los pacientes se los sometan a pruebas de imagen como ecografía y resonancia magnética. La RM es la de elección, porque no utiliza radiación y evalúa los riñones en toda su anatomía.

\section{Bibliografía}

1. Milanés Roldán M.T., Fernández Delgado N., Fundora Sarraff T., Jaime Facundo J.C., Hernández Ramírez P. Hemoglobinuria paroxística nocturna: Actualización. Rev Cubana Hematol Inmunol Hemoter [Internet] 2003 Abr [citado 2017 Abr 02]; 19(1).

2. Cruz, V.J., Hill, A, López, M., Ispizua, A., Schrezenmeier, H., Muus, P., et al. Revista informativa dedicada a la enfermedad Hemoglobinuria Paroxística Nocturna y su Asociación de pacientes 2012; (3): 4-29.

3. Ministerio de Salud Pública del Ecuador. Acuerdo ministerial de 1829 enfermedades consideradas catastróficas. 2012; № 1829.

4. Urbano, I. I., Gaya, A., Colado, E., López, M., Vicente, V., Alberto, O., et al. Diagnóstico y tratamiento de la hemoglobinuria paroxística nocturna. Med Clin 2012, 136 (3): 121-127.
5. Ju, Y. J., Seung, H. K. \& Ha, J. L. Atypical Low-Signal Intensity Renal Parenchyma: Causes and Patterns. Radio Graphics 2002; 22 (4): 833-846.

6. Cruz, V. J. \& Mercedes, L. Asociación de Hemoglobinuria Paroxística Nocturna, Revista HPN 2014; 32 (5): 4-11.

7. Ruiz, L. J., Abraldes, J. A., Artola, T., \& Aguado, M. J. Hemoglobinuria paroxística nocturna: Como sospecharla en nefrologia. Nefrología XIX. 2009: 280-281.

8. Tanaka, Y., Anno, I., Itai, Y. \& Abe, T. Paroxysmal nocturnal hemoglobinuria: MR findings. PubliMed 2005; 17(5): 749-753.

9. Suzukawa, K., Ninomiya, H., Mitsuhashi, S., Anno, I., Nagasawa, T. \& Abe, T. Demonstration of the deposition of hemosiderin in the kidneys of patients with paroxysmal nocturnal hemoglobinuria by magnetic resonance imaging. Intern Med 1993; 32(9): 686-690.

10. Aguirre, L. E., Ballester, P. S. \& Pardo, A. S. Tratamiento de la hemoglobinuria paroxística nocturna: revisión bibliográfica y estudio de utilización del eculizumab. Salud Madrid 2015; 22: 5-11.

11. Sánchez, A. J., Marne. S. D. \& Ruiz, A. M. Hipótesis profesional de la Hemocromatosis. Med Segur Trab (Internet) 2010; 56 (219): 165-185.

12. Sinan, M. D., Karakus, A., Ekmen, M. O., \& Orhan Ayyildiz. Presentation and Management of Paroxysmal Nocturnal Hemoglobinuria: A Single-Center Experience. PMC. Hematol Rep. 2016; 8(1).

13. Mondragón, C. Á., Rojas, M. Johana., Jiménez, C. C. \&, Umaña, G. H. Hemoglobinuria Paroxística Nocturna: reporte de un caso. Inv Andina 2013; 26(15): 716-723. 